\title{
Correction to: DeepDist: real-value inter-residue distance prediction with deep residual convolutional network
}

Tianqi Wu ${ }^{1+}$, Zhiye Guo ${ }^{1+}$, Jie Hou ${ }^{2}$ and Jianlin Cheng ${ }^{1 *}$ (1)

The original article can be found online at https://doi. org/10.1186/s12859-02103960-9

${ }^{*}$ Correspondence: chengji@missouri.edu

†Tianqi Wu and Zhiye Guo have equal contributions to this work

${ }^{1}$ Electrical Engineering and Computer Science Department, University of Missouri, Columbia, MO 65211, USA

Full list of author information is available at the end of the article

\section{Correction to: BMC Bioinformatics (2021) 22:30 https://doi.org/10.1186/s12859-021-03960-9}

Following the publication of the original article [1], the authors identified that the grant of the funding note is incorrect. The correct funding note is given below.

Funding note

Research reported in this publication was supported in part by two NSF Grants (DBI 1759934 and IIS1763246), a DOE grant(DE-SC0021303) and an NIH Grant (R01GM093123) to JC. The funding agencies did not play a role in this research.

The original article [1] has been corrected.

\begin{abstract}
Author details
${ }^{1}$ Electrical Engineering and Computer Science Department, University of Missouri, Columbia, MO 65211, USA. ${ }^{2}$ Department of Computer Science, Saint Louis University, St. Louis, MO 63103, USA.
\end{abstract}

Published online: 29 June 2021

Reference

1. Wu et al. BMC Bioinformatics (2021) DeepDist: real-value inter-residue distance prediction with deep residual convolutional network (2021) 22:30 DOI: https://doi.org/10.1186/s12859-021-03960-9

\section{Publisher's Note}

Springer Nature remains neutral with regard to jurisdictional claims in published maps and institutional affiliations. 